\title{
Percepción de las familias sobre la violencia juvenil, el caso de la Colonia San Francisco de Comayagüela
}

\author{
Marlen Yesenia Jiménez Martínez ${ }^{1}$ \\ Juana Castilla Jiménez ${ }^{2}$
}

\section{RESUMEN}

El presente estudio describe la percepción de las familias de la Colonia San Francisco de Comayagüela sobre la violencia juvenil, tomando en cuenta que la familia es considerada como un sistema dinámico viviente sometido a un continuo establecimiento de reglas y búsqueda de acuerdo a las mismas, en donde sus miembros inician su proceso de desarrollo e interacción con las demás personas. Como parte de la sociedad, las familias están expuestas a un sinnúmero de situaciones que contribuyen a su desarrollo, lo cual puede ser positivo o negativo, tal es el caso de la importancia de conocer a través de sus percepciones qué significa para las familias la violencia, principalmente la juvenil, como un fenómeno que a nivel de país se enfrenta como una problemática que pone en situación de vulnerabilidad principalmente a la niñez y juventud.

Esta es una investigación de tipo cualitativa, con alcance descriptivo de corte transversal, con una muestra intencional. Para conocer las percepciones de las familias sobre la violencia juvenil se analizaron las variables definidas en el marco teórico y operacionalización de variables, su unidad de análisis fueron las familias y para el desarrollo de la investigación se contempló, para datos cualitativos, la técnica de la entrevista semiestructurada. Se contó con una muestra de 28 personas distribuidas en 4 familias nucleares, 4 extensivas y 2 monoparentales: todas pertenecientes a los 8 sectores que conforman la Colonia, que de acuerdo con la información obtenida de la misma, cuatro de estos sectores son considerados puntos rojos o puntos de encuentros de jóvenes.

${ }^{1}$ Beneficiaria de una beca de estudiante de posgrado la DICYP, Posgrado Latinoamericano en Trabajo Social, Facultad de Ciencias Sociales, UNAH: jimenezmartinez76@yahoo.com

${ }^{2}$ Asesora, Posgrado Latinoamericano en Trabajo Social, Facultad de Ciencias Sociales, UNAH: platsunah@yahoo.es 
En cuanto a los hallazgos es importante decir que las familias que formaron parte de la muestra de estudio, dentro de sus percepciones, consideran que la falta de comunicación, la violencia manifiesta en todas sus representaciones, el desconocimiento e irrespeto de los derechos de las familias, la desintegración familiar, la carencia de la práctica de valores como el respeto, influyen en cuanto a que se tornen los espacios familiares y comunitarios violentos, ya que estos escenarios son diversos, en los cuales se desarrollan las personas, influyendo en su desarrollo pleno.

Palabras claves: familia, violencia, derechos humanos, vida cotidiana, poder.

\section{ABSTRACT}

The present study describes the youth violence perception of the families living in San Francisco neighborhood in Comayagüela, taking in consideration the perception of family as a living dynamic system subject to continuous establishment of rules, where its members begin their process of development and interaction with others. As a part of society, families are exposed to a number of situations that contribute positively or negatively to its development, therefore the importance of understanding their perception on youth violence, which puts vulnerable primarily to children and youth in Honduras.

This is qualitative type research, with a cross section descriptive scope, using a sample on purpose. A set of variables defined in the research framework were operationalized, each family was considered as a unit of analysis. A semi-structured interview was contemplated for production work qualitative data. The interviewed sample consisted of 28 people in 4 nuclear families, 4 extended families and 2 single parent families, belonging to the 8 sectors in the neighborhood. The information recollected in the interview indicated four of these sectors considered as points of youth meetings.

The findings portrayed the following perceptions: lack of communication, violence manifested in all its representations, ignorance and disrespect of family's rights, family breakup, lack of values, such as respect. All of these perceptions have a violent influence in family and community spaces, even in different scenarios, influencing their full development as a grown person.

Keywords: family, violence, human rights, everyday life, power 


\section{INTRODUCCIÓN}

Según González (2007), para la teoría de sistemas, la familia es considerada como un sistema dinámico viviente sometido a un continuo establecimiento de reglas y de búsqueda de acuerdo a las mismas. Asimismo, constituye un sistema abierto que pertenece a los grupos primarios en donde se caracteriza por suponer una asociación y cooperación íntima, cara a cara, y por ser fundamental en la formación de la naturaleza social y los ideales del individuo.

En tal sentido, el presente artículo tiene como objetivo dar a conocer la percepción que tienen las familias sobre la violencia juvenil, en el caso de la Colonia San Francisco de Comayagüela, municipio del Distrito Central, departamento de Francisco Morazán, para lo cual fue fundamental considerar cinco categorías: derechos humanos, vida cotidiana, familia, poder y violencia.

El artículo arroja resultados sobre la percepción que tienen las familias de la Colonia San Francisco de Comayagüela sobre la violencia juvenil y su importancia en la dinámica familiar; tal información permitió identificar cómo este fenómeno es considerado y de qué manera repercute en las familias y en el entorno en su conjunto.

Los datos obtenidos permitieron la obtención de resultados que se enfocan en un punto central, que es la pregunta de investigación planteada como investigadora: ¿Cuál es la percepción de las familias sobre la violencia juvenil?

Como aporte social se espera que los hallazgos de esta investigación y las recomendaciones sirvan de manera significativa para conocer cómo las familias ven y viven ante la situación de violencia, ya que al saber sobre esta permite contribuir a mejorar la calidad de vida de las mismas y su entorno, ya que constantemente este sector de la sociedad demanda el respeto a sus derechos y condiciones dignas de vida con seguridad integral, así como también exigen a los Gobiernos a través de políticas públicas y programas que los beneficien en este sentido.

Como aporte académico brinda elementos que contribuyen a una reflexión teórica sobre el objeto de estudio y a la generación de datos e información sobre la temática, la cual puede ser una herramienta de apoyo para profundizar en futuras investigaciones relacionadas con la percepción que tienen las familias sobre la violencia juvenil, ya que en el país esta situación ha sido un fenómeno a veces invisibilizado 0 visto como sucesos cotidianos normales y los estudios en torno al mismo, teniendo como 
parte fundamental las percepciones familiares, son escasos, por eso la importancia que se considera en la investigación.

\section{MÉTODOLOGÍA}

La investigación "Percepción de las familias sobre la violencia juvenil en el caso de la Colonia San Francisco de Comayagüela", es de tipo cualitativa, de carácter descriptiva, de corte transversal, con una muestra intencional.

Para realizar el estudio, la unidad de análisis fueron las familias, el listado de las mismas se obtuvo de la base de datos facilitada por el Proyecto Ampliando Oportunidades, ejecutado por la Cruz Roja Hondureña en la comunidad. Para la selección de la muestra, el universo fue 813 familias de tres tipos: nucleares, conformadas por un total de 281 hogares, lo que equivale a un $34.56 \%$; extensivas, con 308 hogares, equivalentes a un $37.88 \%$ y monoparentales, con 224 hogares que corresponden a un $27.55 \%$; distribuyendo la muestra en los 8 sectores que la comunidad, que de acuerdo con la información obtenida sobre la zona, cuatro de estos son considerados puntos rojos o puntos de encuentros de jóvenes.

La muestra fue de 10 familias, de las cuales se tomaron integrantes de cada grupo familiar conformados por jóvenes, la cual se distribuyó de la siguiente manera: 4 familias nucleares, 4 extensivas y 2 monoparentales. En el trabajo de campo se decidió, del total de familias, entrevistar a 2 familias extensivas, 2 nucleares y 1 monoparental en los sectores considerados como puntos de encuentro de jóvenes y el resto de las entrevistas en los otros sectores de la comunidad.

Dada la importancia de conocer la percepción de las familias sobre la violencia de acuerdo al sector al que pertenecen, identificando la diferencia o similitud en cuanto a su percepción, los criterios de selección de las familias fueron: edad, tipo de familia y nivel educativo de las mismas.

El instrumento de recolección de información utilizado fue la entrevista semiestructurada, realizándose la prueba de instrumentos en una colonia con similares características a la del objeto de estudio. Para el ordenamiento de datos obtenidos en las entrevistas semiestructuradas aplicadas, se desarrolló un proceso de digitalización y ordenamiento de datos en Microsoft Word y Excel. 
Para desarrollar el presente estudio fue de vital importancia contar con información en materia de familia y violencia desde un contexto nacional, hasta el caso específico de la Colonia San Francisco de Comayagüela, permitiendo ampliar la base de la investigación en la temática de la percepción de las familias sobre la violencia juvenil.

\section{ANÁLISIS}

Para el desarrollo de este estudio fue importante conocer algunas características demográficas de la población y familias del país, también es importante mostrar aspectos de la situación real que presenta este sector específico de la población.

\section{Marco contextual}

Para el año 2012, según datos de población del INE (2014), Honduras tenía una población de 7, 935, 846 personas y, de estas, la población masculina es mayoría, con 3, 969, 421 hombres, lo que supone el $50.01 \%$ del total; frente a las 3, 966, 425 mujeres que son el $49.98 \%$.

Honduras se encuentra en la 95 posición de la tabla de población, compuesta por 182 países, y presenta una moderada densidad de población de 71 habitantes por $\mathrm{km}^{2}$. El PIB per cápita es un buen indicador de la calidad de vida y en el caso de Honduras, en 2012, fue de 1,812 euros; por lo que se observa que con esta cifra está en la parte final de la tabla, en el puesto 126, sus habitantes tienen un bajo nivel de vida con relación a los 181 países (INE, 2014).

Tegucigalpa es la capital de la república y se ubica en el departamento de Francisco Morazán, junto a la ciudad gemela Comayagüela forma parte del denominado municipio del Distrito Central. La ciudad se ubica en el centro del país, en un altiplano a unos $990 \mathrm{msnm}$, rodeada de colinas, en donde se asientan principalmente colonias y barrios marginales que surgen por las grandes inmigraciones de población proveniente de diversas zonas rurales de Honduras. Según los resultados de la encuesta permanente de hogares del INE (2013), se estima en 1, 863,291 el número de viviendas en el país y en ellas se albergan 1, 898,966 hogares con 8, 535,692 personas; promediándose una relación de 4.5 personas por hogar a nivel nacional. La cantidad de personas que integran los hogares rurales es mayor que la de los hogares urbanos, 4.7 y 4.3 personas, respectivamente. 
Según el Informe de Desarrollo Humano para Honduras (2011), Honduras continúa siendo un país altamente inequitativo y la inequidad económica está aumentando. Según este Informe, el coeficiente de Gini del país, que mide la inequidad de ingresos, fue de 0.58 en el 2011, lo cual lo ubica como uno de los más altos de América Latina, solo superado por Colombia y Haití.

Al respecto, Muggah citado por Brender (2012), menciona que: "Siendo los escenarios del individuo diversos, en cuanto a la violencia y el sujeto argumenta que por un lado la urbanización es una fuerza que ayuda al desarrollo progresivo de los pobres $y$, por otro lado, aumenta la inseguridad permanente entre los pobres de la urbe" (p.2). Briceño León (2002), en cuanto a la violencia, dice que:

En el siglo XXI no hay guerras en América Latina, sin embargo, las muertes por la violencia causan tantos muertos, mujeres viudas, niños y niñas huérfanas, como en enfrentamientos armados que son mostrados por la televisión, que suceden en diferentes partes del mundo, siendo la violencia una de las primeras causas de muerte entre las personas jóvenes y productivas en edades de entre 15 y 44 años (p. 34).

De acuerdo al Instituto Universitario de Democracia, Paz y Seguridad de la Universidad Nacional Autónoma de Honduras (IUDPAS, 2013), entidad que realiza el Informe del Observatorio de la Violencia: "Durante el mes de enero a junio del 2013 se produjeron un total de 4,993 muertes ocurridas en Honduras con una reducción de $8.8 \%$, es decir, 482 muertes menos en relación al mismo periodo del 2012" (p.1).

Los datos del IUDPAS reflejan que el homicidio sigue siendo la principal causa de muerte violenta con 3,547 muertes, las que representan el $71 \%$ del total reportado; esta categoría representa una disminución del 1.8 \% con relación al año anterior. La segunda categoría más frecuente son los eventos de tránsito con 637 muertes (12.8\%); con relación a los datos del primer semestre de 2012, estas muertes han tenido un aumento del $0.3 \%$. La tercer categoría la ocupan las muertes indeterminadas con 429 víctimas (8.6\%), representando una disminución del $40.7 \%$ con relación al 2012. Por su parte, la violencia no intencional presenta 222 muertes (4.4\%) y un 30 \% de reducción con relación al año anterior, se sigue teniendo una reducción de los homicidios en los primeros seis meses de 2013 (p.1).

La Cruz Roja Hondureña (2010) menciona que:

La inseguridad ciudadana es una de las mayores preocupaciones de la población de Tegucigalpa y Comayagüela, que se ha visto incrementada con la violencia generada 
principalmente por pandillas juveniles y grupos armados relacionados con el crimen organizado y narcotráfico. La proliferación de estas pandillas de jóvenes, conocidas como "maras", se ha convertido en un grave problema de seguridad nacional, local y familiar (p.6).

De acuerdo al Distrito Policial 1-7 de la Colonia San Francisco de Comayagüela, en cuanto a los reportes de incidencia de violencia en la comunidad, en el periodo comprendido entre enero a junio del año 2013 , los casos reportados más frecuentes son las denuncias por escándalo en vía pública, principalmente debido al enfrentamiento entre miembros de barras deportivas del Olimpia y Motagua; en menor caso por estado de ebriedad y peleas varias; con un total de 170 personas reportadas por este motivo, las cuales están entre las edades de 6 a 60 años: de 6-12 años, 1 caso reportado; de 13-18 años, 32 casos; de 19-35 años, 110 casos; de 36-59 años, 26 casos y un caso registrado en edad de 60 años y más; las personas reportadas pertenecen al sexo masculino.

En cuanto a denuncias por violencia doméstica e intrafamiliar, las mismas de acuerdo a la policía local no son reportadas, ya que solo se registraron en este periodo de tiempo 11 casos, intentos de homicidio 1 caso, accidentes de tránsito 1 caso, robos 1 , consumo de drogas 1 caso y 1 caso reportado por violación a una menor de edad (Distrito Policial 1-7, 2013).

\section{Colonia San Francisco}

Está ubicada en el sector noroccidental de Tegucigalpa, con una población aproximada de 3,911 habitantes y con 804 hogares. Pertenece al departamento de Francisco Morazán: limita al norte con el bulevar Fuerzas Armadas y la Residencial Centroamérica; al sur con la represa Los Laureles; al este con la Colonia El Progreso y la Venezuela; al oeste con el Cementerio Santa Anita y la Colonia Israel Norte. Está conformada por 8 sectores: Los Posos, El Rosario, Vuelta del Molino, Cabañas, El Retiro, El Plan, sector 7 de La Popular, sector 8 Kennedy; de los cuales son considerados como conflictivos los sectores: El Plan, La Popular, El Retiro y el sector Kennedy (Cruz Roja Hondureña, 2010).

En la comunidad, en el $57.90 \%$ de los hogares los jefes de hogar son hombres y el $42.10 \%$ mujeres. De acuerdo con el análisis realizado por PAO/CRH (2010), la colonia se caracteriza por considerarse en vías de desarrollo y cuenta con los servicios básicos (agua potable, luz eléctrica, alcantarillado, teléfono, transporte urbano, tren de aseo, internet). Como principales problemas se identifican la disfuncionalidad 
familiar, la inseguridad por la incidencia de grupos de maras y pandillas, influencia del narcomenudeo, jóvenes y adultos involucrados en el sicariato; niños, niñas, jóvenes, mujeres y hombres en situación de calle.

\section{Proyectos en la comunidad}

Entre las acciones que promueven la cultura de paz y el respeto de los derechos humanos, así como la inserción de jóvenes y familias en espacios socioeducativos y ocupacionales que se realizan en la Colonia San Francisco de Comayagüela, se encuentran las del Proyecto Ampliando Oportunidades, el cual es impulsado por la Cruz Roja Hondureña, que se ejecuta a partir del año 2003 en la comunidad. Este surge para el fortalecimiento de la salud urbana y la prevención de la violencia juvenil con población en riesgo social de la Colonia San Francisco y zonas aledañas, centra sus acciones en la generación y fortalecimiento de capacidades humanas a nivel individual, familiar y comunitario, contribuyendo a mejorar la calidad de vida de los jóvenes en riesgo social (PAO/CRH, 2010).

Otra organización fundamental que ha realizado acciones en la comunidad es la Asociación Cristiana de Jóvenes (ACJ), con su oficina central en Tegucigalpa, fundada en 1990 en Honduras. En 1994 inició el Proyecto de Niñez en la Colonia San Francisco de Comayagüela, con el apoyo de YCARE (ACJ, 2013). En su propuesta 2014 para ejecutar en la Colonia San Francisco, contando con una sede ludoteca comunitaria en la zona, ACJ se propuso los siguientes ejes programáticos planificados para contribuir con el fortalecimiento de acciones de desarrollo y juventud:

1. Salud mental y deporte: promueve a jóvenes para que participen en diferentes disciplinas a través de las diferentes federaciones deportivas de Honduras. 60 jóvenes participando en 2 campamentos juveniles organizado por ACJ Honduras.

2. Formación ciudadana: con el Proyecto Escuela de Informática y Ciudadanía, desarrollando procesos en el área de computación y formación ciudadana.

3. Incidencia política: jóvenes capacitados en temáticas relacionadas a incidencia política involucrándose en la organización y ejecución de campañas de limpieza y contra el VIH/sida.

4. Emprendedurismo: jóvenes accediendo a la capacitación sobre elaboración de 
planes de negocio y al financiamiento a través del acompañamiento; asimismo, participando en cursos especiales para el fomento del emprendedurismo.

\section{Perspectiva conceptual}

Esta investigación está enfocada en la percepción que las familias tienen sobre la violencia juvenil, la cual se desarrolla desde el enfoque de los derechos humanos, en los cuales se refleja una interrelación entre cinco categorías de análisis:

1. Derechos humanos haciendo énfasis en la vivencia de los derechos de libertad, igualdad y seguridad, en donde se pretende conocer, a través de la lectura de datos, las percepciones de las familias sobre la violencia juvenil, dando respuesta a parte del objetivo general con el análisis de las respuestas a las interrogantes 2.1 a la 2.8, haciendo cruce de acuerdo al tipo de familia, edad, sexo y escolaridad (ver anexo 1$).^{3}$

2. Vida cotidiana con énfasis en las estrategias de convivencia y toma de decisiones, con la respuestas a las interrogantes 3.1 a la 3.3 se hace el análisis para dar respuesta a parte del objetivo de conocer las estrategias que las familias adoptan ante la presencia de la violencia juvenil, cruce de acuerdo al tipo de familia, edad, sexo y escolaridad (ver anexo 1$)^{4}{ }^{4}$

3. En familia incluye las subcategorías: percepciones de la violencia, roles familiares y mecanismos de comunicación, tratando de dar respuesta al objetivo de conocer la percepción que tienen las familias sobre la violencia, el análisis se hace obteniendo las respuestas a las interrogantes 4.1 a la 4.8 , realizando cruce de acuerdo al tipo de familia, edad, sexo y escolaridad (ver anexo 1). ${ }^{5}$

4. En la categoría de poder visualizar las interrelaciones familiares, en donde el análisis de datos se desarrolló dando respuesta a las interrogantes 5.1 a la 5.5, logrando dar respuesta a parte del tercer objetivo, que es conocer la estrategias

\footnotetext{
${ }^{3}$ Las interrogantes 2.1-2.8 hacen énfasis en lo que los entrevistados comprenden en cuanto a los derechos de libertad, igualdad y seguridad, tanto a nivel individual como familiar.

${ }^{4}$ Las interrogantes 3.1 a la 3.3 se refieren a la vida cotidiana, es decir, de cómo las familias toman decisiones, sobre qué temas y estas de qué manera influyen en sus miembros.

${ }^{5}$ Las interrogantes 4.1-4.8 tratan de la familia y su percepción sobre la violencia, esto hace énfasis en la comunicación familiar, cómo se distribuyen las responsabilidades en el hogar, asimismo de los entrevistados se conoce la forma en la que influyen en la distribución de responsabilidades, viéndose estas prácticas como parte de las acciones normales de convivencia o una forma de ejercer la violencia en cualquiera de sus manifestaciones.
} 
que las familias adoptan ante la presencia de la violencia juvenil, realizando cruce de acuerdo al tipo de familia, edad, sexo y escolaridad (ver anexo 1$)^{6}{ }^{6}$

5. Categoría de violencia, los tipos de violencia, repercusiones de la violencia, mecanismos para generar temor y actos violentos; el análisis de esta categoría se desarrolló de acuerdo a las respuestas a las interrogantes 6.1 a la 6.23, con las cuales se da respuesta al objetivo de conocer las repercusiones de la violencia juvenil en las familias de la Colonia San Francisco y su contexto, realizando cruce de acuerdo al tipo de familia, edad, sexo y escolaridad; de esta manera se reflejaron diferencias y similitudes en las respuestas (ver anexo 1). ${ }^{7}$

Con el análisis de la información se conoce la percepción de las familias sobre la violencia juvenil y cómo esta repercute en la familia, quienes se plantean estrategias para romper las condiciones que les impone la violencia. En cuanto al derecho en su condición de persona, de acuerdo a la Declaración Universal de los Derechos Humanos de la Asamblea General de Naciones Unidas, Resolución 217 (A.III), se:

Establece que la libertad, la justicia y la paz en el mundo reconocen la dignidad intrínseca y derechos iguales e inalienables de todas las y los miembros de la familia humana, los cuales deben ser protegidos por un régimen de derecho, promoviendo un progreso social y elevando el nivel de vida dentro de un concepto más amplio de la libertad (Oficina del Alto Comisionado de los Derechos Humanos, 1948).

De igual manera, el Informe de Desarrollo Humano (2000) señala que:

Tanto el desarrollo humano como los derechos humanos comparten un propósito y una visión en común que es el de garantizar la libertad, el bienestar y la dignidad de las personas, es por ello que para el desarrollo humano, los derechos humanos van de manera intrínseca en el desarrollo y este como un medio para que los derechos humanos sean una realidad, mismos que se traducen en la protección y vigilancia evitando en la medida de lo posible en los individuos aflicción y el dolor, construyéndose como actor

${ }^{6}$ En las interrogantes 5.1-5.5 se escudriña la relación de poder: la importancia de las relaciones interpersonales entre los miembros de la familia, las cadenas de mando y cómo estas influyen en su dinámica familiar, tomando en cuenta las estrategias que les permitan hacer frente a la violencia.

${ }^{7}$ Las interrogantes 6.1-6.23 hacen énfasis en la violencia en sus diferentes representaciones, las familias dan a conocer cómo es considerada la violencia de acuerdo a su percepción personal, de qué manera afecta la misma, a sus miembros corno a su entorno, quiénes son los generadores de esta, principalmente en la comunidad, también se hace mención por parte de los entrevistados sobre la existencia de programas y proyectos que promuevan la cultura de paz y acciones integrales en prevención de la violencia. 
capaz de modificar su ambiente y de hacer sus experiencias de vida, pruebas de su libertad (p. 6-7).

Desde el enfoque de los derechos humanos se sitúa a las personas como la prioridad principal en el desarrollo: "Desde el punto de vista normativo está basado en las normas internacionales de derechos humanos y desde el punto de vista operacional está orientado a la promoción y protección de los derechos humanos" (IDH, 2000, p.23). El mismo analiza las desigualdades que impiden el desarrollo en su conjunto.

En cuanto a la libertad de las personas, Touraine (1995) también menciona que: "En la medida en la que el sujeto se crea, está centrado en sí mismo y ya no en la sociedad, es definido por su libertad y no solo por sus roles, los individuos se convierten en sujeto cuando se liberan de las normas sociales del deber del Estado" (p.182); además, para el autor la idea de sujeto combina tres elementos: "La resistencia a la dominación, el amor a sí mismo y el reconocimiento de los demás como sujetos y el respaldo dado a las reglas políticas y jurídicas que dan al mayor número de personas las mayores posibilidades de vivir como sujetos" (p.182).

En la vida cotidiana es importante conocer cómo los derechos humanos juegan un papel relevante en el desarrollo de las personas en todos sus ámbitos, a lo que Lechner (1990) indica que:

La libertad se vive en la vida cotidiana entendida esta y tomando como punto principal la desigualdad y la dominación de la siguiente manera: tanto ámbito público-privado representa una existencia inferior respecto al mundo público y solo superando el mundo de las necesidades y, por ende, de la dominación y la desigualdad los hombres pueden llegar a realizarse como individuos libres e iguales (p.40).

Además, este autor hace énfasis en que la vida cotidiana es: "Fundamentalmente el campo de análisis de los contextos en los cuales diferentes experiencias particulares llegan a reconocerse en identidades colectivas, donde la subjetividad no nace con el individuo, surge en sociedad y es sostenida por ella" (p.59).

La vida cotidiana de acuerdo a la Declaración Universal de los Derechos Humanos en su Artículo 16, inciso 3, dice que: "La familia es el elemento natural fundamental de la sociedad y tiene derecho a la protección de la sociedad y del Estado", por lo cual como parte de los retos y funcionamiento de las políticas públicas basadas en los derechos humanos, es la protección de la familia en su conjunto con oportunidades y el ejercicio pleno de sus derechos independientemente de su estatus social, tomando 
en cuenta las desigualdades existentes y la dominación en la vida cotidiana de las familias.

Por su parte, González Gallegos (2007) indica que la teoría de sistemas hace mención de que: "La familia es considerada un sistema dinámico, viviente sometido a un continuo establecimiento de reglas y búsqueda; de acuerdo a ellas se considera a la familia como un sistema integrador multigeneracional, caracterizado por múltiples subsistemas de funcionamiento interno e influido por una variedad de sistemas externos relacionados" (p.111-112). De acuerdo a esta teoría, las personas deben ser estudiadas no a nivel individual y aisladas de su medio, sino por el contrario, se debe establecer una relación entre las personas y su entorno y cómo este influye en su interacción social y familiar.

Garciga (2006), al hacer referencia a la familia, reafirma que este sistema dinámico es visto como:

Un grupo social básico creado por vínculos de parentesco o matrimonio presente en todas las sociedades, idealmente proporciona a sus miembros protección, cariño, educación y socialización, la misma cumple funciones importantes en el desarrollo biológico, psíquico y social de las personas asegurando la inserción de las mismas en la vida social, de igual manera la transmisión de valores culturales de generación en generación (p.15).

Lo que coincide con la teoría de sistemas en cuanto a la importancia de ver a las personas como un todo en sociedad, que debe gozar en plenitud de los derechos humanos en su conjunto, visualizando el deber del Estado de garantizarlos y defenderlos.

Conocer sobre los modelos de familia de acuerdo a referencias teóricas es vital tomando en cuenta que ya no se habla solo de la familia nuclear, sino de familias, las que pueden ser construidas o reconstruidas de acuerdo a la situación. En tal sentido, Cruz (2001) indica que la sociología hace mención de los tipos de familias predominantes en la sociedad: nuclear, extensa o consanguínea, monoparental, familia de madre soltera, de padres separados, familias reconstituidas.

Foucault (1979) señala que: "En la interrelación entre las personas ya sea dentro de su núcleo familiar, comunitario, su vida cotidiana y en la sociedad en general, se refleja ese vínculo entre las mismas y el poder y cómo este va creando o no relaciones de igualdad y respeto de los derechos humanos" (p.153). Asimismo enfatiza que: 
Las relaciones de poder desde el ámbito familiar, hasta la sociedad en general, han sido de desigualdad, no estableciéndose una relación entre poder y sexo en la cual definitivamente no hay una relación de representación, sino al contrario, según lo que argumenta las relaciones de poder pueden penetrarse materialmente en el espesor mismo de los cuerpos, sin tener que ser incluso sustituidos por la representación de los sujetos (p.156)... El poder no solo pesa solamente como una fuerza que dice no, sino que de hecho la atraviesa todo el cuerpo social más que como una instancia, como una instancia negativa que tiene como función reprimir (p.38).

Sanmartín (2000) señala que: "Decir que somos agresivos por naturaleza no conlleva a aceptar que por naturaleza somos violentos, no hay violencia sino hay cultura, la misma no es producto de la evolución biológica ni de la bioevolución, como se dice frecuentemente, es más bien resultado de la evolución cultural. La violencia se manifiesta bajo diversos contextos y bajo formas diferentes" (p.3).

Mientras que para Benjamín (1999): "La violencia es considerada un fenómeno histórico enmarcado en un contexto determinado y su dinámica debe ser analizada tanto desde el campo moral definiéndola desde el derecho y la justicia". Para Amartya Sen (2007):

El conflicto y la violencia actuales son sostenidos al igual que en el pasado por la ilusión de una identidad única, por la creencia en el poder abarcador de una clasificación singular que ignora la relevancia de otras formas más allá de las culturas y de las religiones, en la cual los individuos se perciben a sí mismos, por ejemplo la profesión, la clase, la lengua, la moral o la política y el género. Comprendiendo la libertad humana como una manera de combatir el odio confirmado en un poder dominante" (p. 45).

Por otro lado, Manlio Argueta (2006) al hablar sobre la violencia en el estudio hecho para la CEPAL, reafirma la preocupación de que: "Los rostros de la violencia son casi siempre jóvenes, como víctimas y como victimarios". El autor indica que: "La violencia es un derivado lógico de la pobreza" (p.7); pero contrario a lo que podría parecer esta como causa, los mayores grados de violencia no se concentran en las zonas más pobres del continente, sino en aquellos contextos en donde se combinan el deterioro de condiciones económicas, políticas y sociales.

Las familias en sociedad, tomando en cuenta su dinámica en la vida cotidiana y las desigualdades existentes en su interior y en lo externo, en donde hay una lucha de poder, están expuestas a la violencia, la cual se manifiesta de diferentes formas, como se describe a continuación. 
Arriagada (2007) menciona que la violencia doméstica:

En sus diversas manifestaciones es referida a la tortura corporal, acoso y violación sexual, psicológica, limitación de la libertad de movimiento, lo cual es una eminente violación a los derechos humanos, en cuanto a esto la familia es un espacio paradójico siendo el lugar de afecto e intimidad, pero al mismo tiempo un lugar privilegiado para el ejercicio de la violencia (p.110).

La violencia juvenil aparece como un fenómeno de la violencia escolar, en las múltiples peleas que se forman en las zonas de ocio, en las conductas agresivas que acompañan al tráfico y consumo de drogas, actos vandálicos, peleas entre bandas juveniles, acosos sexuales entre jóvenes; estos actos son realizados por los jóvenes, quienes suelen afectar a otros jóvenes, violencia que inició de esta manera y que se ha expandido a diferentes manifestaciones en las comunidades.

De acuerdo a Polaino Lorente (2008): "La violencia juvenil está íntimamente ligada a la violencia intrafamiliar donde los mecanismos de aprendizajes son evidentes de los padres entre sí hacia los y las hijas y estos replicando la misma como un mecanismo de aprendizaje, la violencia intrafamiliar igualmente es un reflejo de la violencia social en cuyas redes está asentada la familia" (p. 240).

Estas formas de violencia son causantes de terror y miedo en las familias y su vida cotidiana, como lo dice Mannoni (1987): "El miedo es una experiencia universal, el cual comienza a temprana edad, o sea que las personas no están frente al miedo, sino que siempre están en constante miedo potencial y su presencia queda indicada por la prudencia con la que actuamos" (p. 6); de acuerdo con esto, las familias en su conjunto pueden someterse al miedo y a la violencia o dar respuesta de la misma manera.

\section{CONCLUSIONES}

1. Al analizar la situación del contexto de las familias de la Colonia San Francisco en este estudio, se pudo constatar la situación real en la que se encuentran, es decir, su vulnerabilidad ante el fenómeno de la violencia juvenil; así, dentro de las estadísticas de la policía local se evidencia que los jóvenes, principalmente organizados en barras de equipos deportivos, promueven la violencia. 
2. Al ver los datos de país en cuanto a la violencia, se refleja que esta es creciente, lo que debe ser una preocupación para el Estado de Honduras, dado que a través de las políticas públicas en familia y seguridad, principalmente, debe ser el garante de los derechos humanos de las personas.

3. Con relación a las acciones que el Estado de Honduras ejerce a favor de las familias, se refleja de acuerdo a la percepción que las familias tienen sobre los derechos humanos y la violencia, que el Estado no garantiza en su totalidad la protección y el respeto de los mismos; en cuanto a los miembros del hogar, tanto los jóvenes como las personas adultas en su mayoría desconocen el reconocimiento de sus derechos, lo mismo manifestaron en cuanto a los derechos de libertad, igualdad y seguridad, la carencia de la práctica y reconocimiento de los mismos.

4. Las percepciones de las familias consideran que la falta de comunicación, la violencia manifiesta en todas sus representaciones, el desconocimiento e irrespeto de los derechos de las familias, la desintegración familiar y la pérdida de valores, como el respeto, influyen en que se tornen los espacios familiares y comunitarios violentos, ya que estos escenarios en los que se desarrollan las personas son diversos y esto influye en su pleno desarrollo.

5. En cuanto a la violencia, en el caso de Honduras dentro de las políticas públicas se incluye la política nacional de la prevención de la violencia que afecta a la infancia y a la juventud; los destinatarios son los integrantes de las familias, dado que la violencia repercute en sus ámbitos de desarrollo individual y colectivo. Producto de los testimonios se puede decir que la violencia, independientemente de quien la genere, afecta a las familias, sus jóvenes y la estabilidad de los vecinos; por ejemplo, en sectores que dentro de la Colonia San Francisco son considerados tranquilos, los informantes señalaron que son lugares de encuentro de jóvenes que vienen de otros lados a generar violencia con golpes, gritos, amenazas y muertes. En consecuencia, se considera pertinente que a través de políticas ratificadas y pactos internacionales se busquen alternativas que contrarresten esta violencia juvenil en el país y, por ende, en las comunidades más vulnerables ante este fenómeno.

6. Es importante conocer si realmente el Estado, a través de la policía local, controla la violencia y delincuencia generada por jóvenes, o si son los grupos de jóvenes los que imponen su poder controlando las comunidades y generando violencia; de igual manera, indagar si sucede lo mismo al interior de los hogares. 
7. En cuanto a los grupos que los informantes distinguieron como generadores de violencia en la comunidad, fueron principalmente los de jóvenes que integran barras deportivas, principalmente la Revo y la Ultra, las cuales se pelean territorio y reclutan jóvenes para cometer actos violentos entre sí, afectando a otras personas de la comunidad; sin embargo, también mencionaron el grupo de maras como un grupo controlador, las drogas y el alcohol como parte de la forma de operar de estos grupos, anteponiendo sus propias políticas de seguridad.

8. En cuanto a las estrategias para la violencia, definidas por las familias desde su vida cotidiana, se dan vínculos de poder en el matriarcado, en el caso de las familias extensivas y nucleares entrevistadas; no ocurre así en las nucleares, en las cuales en unas el mando es compartido entre padres y madres y en otras el mando solo es patriarcal, considerando algunos de sus integrantes que el poder en la familia es normal para mantener el orden de sus miembros; sin embargo, algunos consideraron que la forma de mando genera conflictos al interior de las familias y esto contribuye a que se genere violencia por los desacuerdos entre sí.

9. Entre las estrategias de las familias, aparte del poder a cargo de madres y padres, está el vincularse a grupos de la iglesia, juveniles y sociales dentro de la zona. Por ejemplo, algunas familias reconocieron la existencia de proyectos sociales como la Asociación Cristiana de Jóvenes (ACJ) y el Proyecto Ampliando Oportunidades de la Cruz Roja Hondureña, como instituciones que contribuyen a la erradicación de la violencia en la comunidad a través de espacios recreativos, culturales, ocupacionales y de acompañamiento familiar, los cuales promueven el desarrollo pleno de las personas por medio de sus acciones.

10. Las familias y la comunidad son espacios fundamentales dentro de la sociedad para el desarrollo de las personas, tanto adultos como jóvenes y niños, ya que todos son miembros de una sociedad en la cual la violencia se acrecienta en sus diferentes formas, por lo que las estrategias ante la presencia de la violencia son básicas y fundamentales e implican la puesta en marcha de acciones integrales y solidarias que contribuyan a revalorizar el rol de los jóvenes y sus familias en la sociedad. 


\section{AGRADECIMIENTOS}

A la Dirección de Investigación Científica y Posgrado de la UNAH por el financiamiento otorgado para la realización del presente estudio. También a Corina Hernández, directora del PLATS; a mi asesora Juana Castilla y al personal docente y administrativo del Posgrado Latinoamericano de Trabajo Social de la UNAH por su apoyo y orientación durante este proceso de enseñanza-aprendizaje.

\section{BIBLIOGRAFÍA}

Arguello Rodríguez, Manuel. (1990). Desarrollo urbano. Costa Rica: EUNED.

Arriagada, Irma. (2007). Familias y políticas públicas para América Latina, una historia de desencuentros. Santiago de Chile: CEPAL.

Banco Mundial. (2011). Crimen y violencia en Centroamérica, un desafío para el desarrollo. Departamentos de Desarrollo Sostenible y Reducción de la Pobreza y Gestión Económica, Región de América Latina y el Caribe.

Barg, Mag Liliana. (2006). Familia: un campo para el trabajo social. Argentina: Universidad de Buenos Aires.

Barret, Michelle y Mclntosh, Mary. (1995). Familia vs. Sociedad. Bogotá: Tercer Mundo Editores.

Brender, Natalie. (2012). Resumen investigando el dilema urbano, urbanización, pobreza y violencia, basado en el estudio de Robert Muggah. Canadá: Centro Internacional de Investigaciones para el Desarrollo de Canadá.

Briceño León, Roberto. (2002). La nueva violencia urbana de América Latina. Sociologías Porto Alegre Brasil, 4(8), 34-55. Brasil.

Cerruti, Marcela y Binstock, Georgina. (2009). Familias latinoamericanas en transformación. Desafíos y demandas para la acción pública. Santiago de Chile: CEPAL.

Centro de Investigación y Promoción de los Derechos Humanos. (2003). Los derechos económicos, sociales y culturales: Reflexiones sobre su concepto y situación en Honduras. Tegucigalpa.

Cruz, Antonio. (2001). Sociología: una desmitificación, guía de estudio, sociología de la desmitificación. España: CLIE.

Dammert, Lucía y Arias, Patricia. (2007). El desafío de la delincuencia en América Latina. Santiago de Chile: CEPLAN.

Durkheim, Emile. (2001). La división del trabajo social. España: AKAL.

Distrito Policial 1-7, Colonia San Francisco. (2013). Registros estadísticos de reportes 
de incidencias de la Colonia San Francisco de Comayagüela, de enero a junio de 2013.

Equipo de Reflexión Investigación y Comunicación, ERIC. (2005). Maras y pandillas en Honduras. Honduras.

Flores Fonseca, Manuel Antonio. (2003). Hogares y familias en Honduras. Tegucigalpa: UNAH.

Foucault, M. (1979). Micro física del poder. España: Piqueta Seseña.

Garciga Ortega, Octavio. (2006). Estilos de vida saludable. Tegucigalpa: Editorial Peniel.

González Gallegos, Juan Max. (2007). La familia como sistema. Revista Paceña, 4(6), 111-114.

Instituto Nacional de Estadísticas. (2013). Cuadragésima cuarta encuesta permanente de hogares de propósitos múltiples. Honduras.

Instituto Hondureño de la Niñez y la Familia (2010). Niñez y adolescencia en el Plan de Nación: Propuesta de inclusión del tema infancia en el Plan de Nación, inclusión y el marco jurídico nacional e internacional. Honduras.

Letchner, Norbert. (1990). Los patios inferiores de la democracia. Chile: Fondo de Cultura Económica.

Manfred, Liebel. (1992). Maras y pandillas en Centroamérica, señas de identidad. Perú: Universidad Técnica de Berlín.

Mannoni, Maud y Potchar, Ricardo. (1987). El niño, su enfermedad y los otros. Argentina: Nueva Visión.

Mejía Herrera, Bárbara Isabel. (2004). Crisis de institucionalidad en la atención de riesgo social de adolescentes en Honduras. Tesis. Universidad Nacional Autónoma de Honduras. Tegucigalpa.

Polaino Lorente, Aquilino. (2008). Violencia juvenil y violencia familiar. España: Díaz de Santos.

Posas, Mario. (2006). Delincuencia, inseguridad ciudadana y desarrollo humano en Honduras. Honduras: Programa de las Naciones Unidas para el Desarrollo.

Programa de Políticas de Juventud, POLJUVE. (2009). Informe para discusión delincuencia juvenil, maras y pandillas. Honduras.

Proyecto Ampliando Oportunidades/Cruz Roja Hondureña. (2010). Propuesta Fase III PAO. Honduras.

Proyecto Ampliando Oportunidades. (2006). Estudio línea base Colonia San Francisco y zonas aledañas. Honduras.

Rietti, Mario. (2008). Plan Nacional de Desarrollo Humano Sostenible 2021. Tercer borrador para promover una cultura de transparencia. Honduras.

Sen, Amartya. (2007). Identidad y violencia. España: KATZ.

Sen, Amartya. (1999). Desarrollo y libertad. España: Planeta. 
Sen, Amartya. (2011). La idea de la justicia. Ensayo de derecho. España: Taurus.

Touraine, Alain. (1995). ¿Qué es la democracia? La limitación del poder. Argentina: Fondo de Cultura Económica.

Touraine, Alain. (1995). ¿Qué es la democracia? Los derechos del hombre contra la soberanía popular. Argentina: Fondo de Cultura Económica.

Touraine, A. (1995). ¿Qué es la democracia? Ciudadanía y comunidad. Argentina: Fondo de Cultura Económica.

Torrico Linares, Esperanza. (2002). El modelo ecológico de Bronfrenbrenner como marco teórico de la psicooncología. España: Servicio de Publicaciones de la Universidad de Murcia. Recuperado de: http/www.la familia y su funcionalidad 


\section{ANEXO 1}

UNIVERSIDAD NACIONAL AUTÓNOMA DE HONDURAS FACULTAD DE CIENCIAS SOCIALES

\section{MAESTRIIA LATINOAMERICANA EN TRABAJO SOCIAL CON ORIENTACIÓN EN GESTIÒN DEL DESARROLLO}

\section{PERCEPCIÓN DE LAS FAMILIAS SOBRE LA VIOLENCIA JUVENIL, CASO COLONIA SAN FRANCISCO DE COMAYAGÜELA}

\section{Entrevista semiestructurada dirigida a familias}

Objetivo de la entrevista: conocer la percepción de las familias de la Colonia San Francisco de Comayagüela sobre la violencia juvenil.

Entrevista \#:

Fecha:

\section{Datos generales del entrevistado}

1.1. Tipo de familia: Monoparental Extensiva Nuclear Estado civil del entrevistado:

1.2 Composición del núcleo familiar:

\begin{tabular}{|c|c|c|c|c|c|c|c|c|c|c|}
\hline No & Nombre & Sexo & & & ngo de & edad & & Parentesco & | Nivel & Ocupación \\
\hline & & & $13-15$ & 16-18 & $\mid 19-35$ & $36-59$ & 60 y mas & & & \\
\hline 1. & & & & & & & & & & \\
\hline 2. & & & & & & & & & & \\
\hline 3. & & & & & & & & & & \\
\hline 4. & & & & & & & & & & \\
\hline 5. & & & & & & & & & & \\
\hline
\end{tabular}




\section{Derechos Humanos}

2.1. ¿Para usted qué son los derechos humanos? Comente.

2.2. ¿Cuáles son los derechos que debemos disfrutar? Comente.

2.3. ¿Ha escuchado hablar del derecho a la libertad? Comente.

2.4. ¿Ha escuchado hablar del derecho a la igualdad? Comente.

2.5. ¿Ha escuchado hablar del derecho a la seguridad? Comente.

2.6. ¿Cree que estos derechos son iguales para la persona, como para la familia? Explique.

2.7. ¿Cuándo considera usted que estos derechos son violados para una persona?

2.8. ¿Cuándo considera usted que estos derechos son violados en una familia?

2.9. ¿Cree que la violación de estos derechos afecta a la persona? Explique.

2.10. ¿Cree que la violación de estos derechos afecta a la familia? Explique.

\section{Vida cotidiana}

3.1. ¿Hay decisiones que se toman en familia?

3.2. ¿Cómo hacen para la toma de decisiones?

3.3. ¿Las decisiones que se toman en familia sobre qué temas son?

3.4. ¿Las decisiones que se toman cómo influyen en la familia? Explique.

\section{Familia}

4.1.¿Cómo es la comunicación en la familia?

4.2. ¿Hay comunicación cuando se distribuyen las responsabilidades en la familia?

4.3. ¿Cómo se asignan esas responsabilidades?

4.4. ¿Se afectan las relaciones familiares cuando se asignan esas responsabilidades?

4.5. ¿Existe respeto entre las y los miembros del hogar? Explique.

4.6. ¿Cómo se dan esas formas de respeto? Explique.

4.7. ¿Influye en las relaciones de las personas en el hogar, el respeto? Explique.

4.8. ¿El cumplimiento de las responsabilidades genera conflicto entre las personas del hogar? Comente.

\section{Poder}

5.1. ¿En esta familia quién manda?

5.2. ¿Cómo es la forma de mandar?

5.3. ¿La forma de mandar trae problemas? Sí No ¿Como cuáles? 
5.4. ¿Usted cree que dentro de una familia, alguien debe mandar? ¿Por qué? Explique.

5.5. ¿La forma cómo esa persona manda interviene las relaciones que se dan entre las personas del hogar? Explique.

\section{Violencia}

6.2. ¿Cuándo escucha sobre la palabra violencia, en qué piensa?

6.3. ¿Con que la asocia?

6.4. ¿Sabe si hay diferentes tipos de violencia? Puede mencionarlos.

6.5. ¿Qué opinión tiene sobre la violencia usted? Comente.

6.5. ¿Cuál ha sido su última experiencia violenta? ¿En dónde y de parte de quién? Describa.

6.6. ¿Considera que en la familia en algún momento se han dado manifestaciones violentas? Comente.

6.7. ¿De qué tipo?

6.8. ¿Qué piensa usted de que en el hogar exista violencia?

6.9. ¿Qué piensa usted de que en la comunidad exista violencia?

6.10. ¿Pasan en la comunidad actos de violencia? Sí__ No___ Cuáles?

6.11. ¿Cuál será la causa?

6.12. ¿Los y las jóvenes de esta comunidad tienen prácticas violentas? Explique.

6.13. ¿Usted considera que existe violencia de estos jóvenes hacia otras personas u otros jóvenes de la comunidad? Explique.

6.14. ¿Eso es frecuente? Sí No Explique.

6.15. ¿Hay lugares específicos en la comunidad donde se realizan esos actos violentos?

6.16. ¿La violencia juvenil se vive en la familia?

6.17. ¿Cómo afecta la violencia juvenil a la familia?

6.18. ¿Qué tipo de medidas adopta la familia cuando la violencia juvenil afecta a sus miembros? Descríbalas.

6.19. ¿En algún momento han buscado algún tipo de apoyo para enfrentar la violencia? Explique.

6.20. ¿La violencia juvenil se vive en la comunidad?

6.21. ¿Como afecta la violencia juvenil la vida comunitaria?

6.22. ¿Qué tipo de medidas adopta la comunidad ante la presencia de la violencia juvenil? Explique.

6.23. ¿Cómo comunidad han buscado algún tipo de apoyo para enfrentar la violencia juvenil? 
